\title{
CONTRIBUTION A L'ÉTUDE \\ D'UNE MÉTHODE D'IDENTIFICATION \\ DES LAITS ET FROMAGES \\ AU MOYEN DE L'ÉLECTROPHORÈSE \\ SUR GEL DE POLYACRYLAMIDE
}

(fin)

par

L. ASSENAT

Laboratoire de la Société des Caves et des Ets. L. Rigal 12-Roquefort

Deuxième partie

\section{ADULTÉRATION DES LAITS ENTRE EUX ADULTÉRATION DES FROMAGES}

Chapitre premier

\section{Adultération des laits}

I) Adultération lait de brebis avec lait de vache.

Le problème des adultérations du lait de brebis avec d'autres laits, et celui de vache en particulier, fait partie des préoccupations de l'industrie de Roquefort. La production du Roquefort est définie par la loi française du 26 juillet 1925, garantissant l'appellation d'origine de ce fromage et sa fabrication à partir de lait de brebis exclusivement.

Ainsi que nous l'avons noté précédemment, les caséines $\alpha_{\zeta}$ des laits de vache et brebis ont des $\mathrm{Rx}$ électrophorétiques nettement différents; si bien que pour un mélange de ces deux laits, on retrouve séparément les deux groupes de protéines.

Pratiquement, on parvient à déceler dans de très bonnes conditions et très sûrement, l'addition de lait de vache à partir de 2 p. 100.

Sur les électrophorèses figure 11, nous avons réalisé des mélanges de lait de vache dans le lait de brebis dans les proportions respectives de 40 p. 100,20 p. 100,10 p. 100,5 p. 100. 


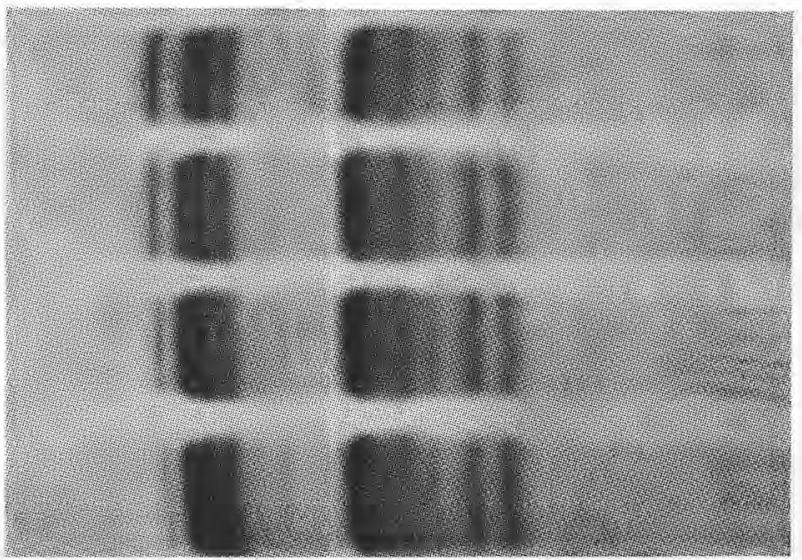

40 p. 100

lait de vache

20 p. 100

lait de vache

10 p. 100

lait de vache

Fig. 11.

Electrophorèse de mélanges : lait de brebis-lait de vache.

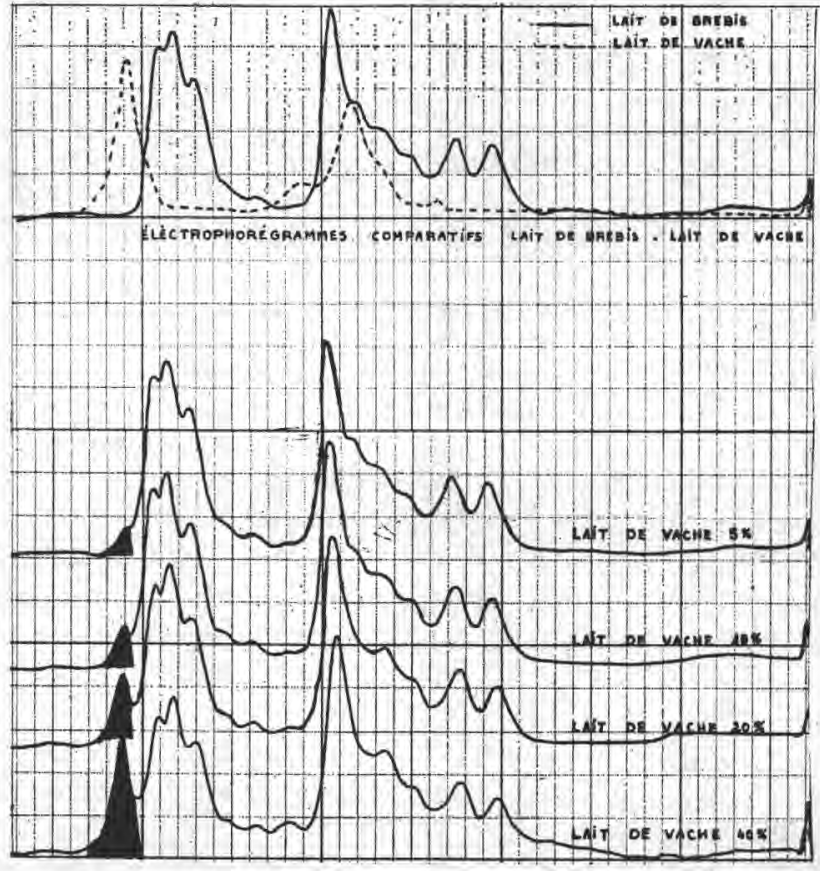

Fig. 12.

5 p. 100

lait de vache

\section{Iig. 12.}

Electrophorégramme de mélanges : lait de brebis-lait de vache. 
On note que les caséines $\alpha_{\zeta}$ vache migrent séparément et nettement au-devant des caséines $\alpha_{\zeta}$ brebis. Si nous évaluons optiquement l'importance des bandes $\alpha_{\varsigma}$ vache (figure 12), nous notons une relation de proportionnalité entre cette densité optique et le pourcentage de caséines de vache mises en œuvre. On obtient donc une droite (figure 13).

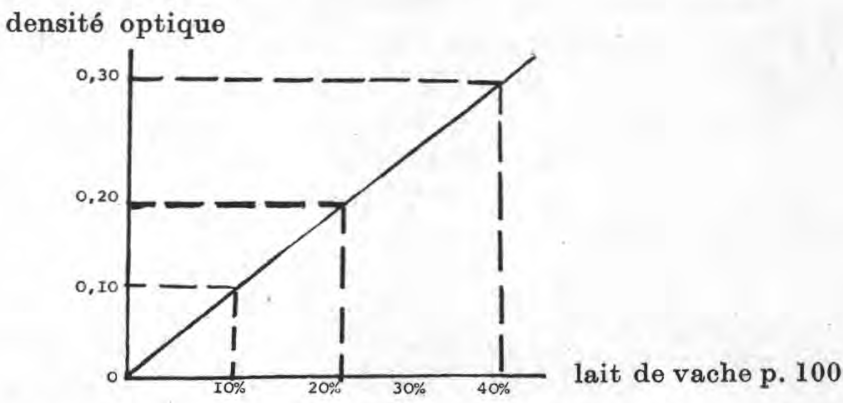

Fig. 13. - Evaluation du pourcentage de lait de vache d'après la valeur densitométrique de la caséine $\alpha_{\zeta}$ vache.

Il est donc théoriquement possible d'évaluer une fraude de cette nature à partir d'un abaque construit sur cette base.

Nous devons cependant considérer que d'un lait à un autre, les variations des taux de matières azotées peuvent atteindre une certaine amplitude. Il n'est donc pas possible de chiffrer les fraudes de façon rigoureuse à partir d'un schéma type moyen; mais nous admettons que cette évaluation est suffisante dans la pratique.

\section{2) Adultération du lait de chèvre avec du lait de vache.}

En France le lait de chèvre a, d'une façon générale, une valeur marchande supérieure à celle du lait de vache. Le problème des fraudes avec le lait de vache peut donc se poser.

Nous savons que les $\mathrm{Rx}$ des caséines $\alpha_{\mathcal{F}}$ vache et chèvre ont un écart un peu plus accentué que dans le cas vache-brebis.

L'identification et l'évaluation de la fraude éventuelle lait de vache sont donc effectuées sur la base de la présence des caséines $\alpha_{\varsigma}$ vache, de la même façon que pour les adultérations de lait de brebis avec le lait de vache.

Dans l'éventualité où le problème inverse se poserait - recherche d'adultération du lait de vache avec lait de chèvre - la base d'identification et d'évaluation serait la caséine $\alpha_{\xi}$ chèvre.

\section{3) Adultération du lait de brebis avec le lait de chèvre.}

Ainsi que nous l'avons observé en première partie, les laits de brebis et de chèvre ont beaucoup de similitudes. 
Les caséines sont peu différentes pour les $\alpha_{\xi}$, sensiblement identiques pour les $\beta$ : reste cependant le paracaséine $x$ qu'il faut isoler avant examen : $\mathrm{Rx}-0,08$. Elle est comme nous l'avons vu caractéristique de chacune de ces deux catégories de lait.

Nous avons également noté que les $\beta$-lactoglobulines chèvre avaient un groupement différent de celui des laits de brebis.

Néanmoins, si ces différences sont suffisantes pour la différenciation de laits purs, les possibilités paraissent plus réduites pour ce qui concerne l'identification des mélanges.

A ce jour, nous pouvons difficilement identifier au-delà de 30 p. 100 de lait de chèvre dans le lait de brebis. Des études ultérieures nous permettront peut-être de tirer un meilleur parti des caractéristiques déjà relevées.

\section{Chapitre II}

\section{Adultération des produits fromagers}

\section{1) Mélange de fromages affinés.}

Nous avons limité cette étude aux mélanges de fromages Roquefort et Bleu de vache. Deux cas peuvent se présenter: ou bien le fromage est fabriqué à partir de mélanges lait de brebislait de vache; ou bien les produits fromagers dérivent directement d'un mélange des fromages Roquefort et Bleu : fromages fondus, dressings, etc.

a) Fromages fabriqués avec mélanges lait de brebis-lait de vache.

Nous avons procédé à des examens électrophorétiques de fromages fabriqués avec différentes proportions des deux laits. Nous avons observé sur les extraits protéiniques de ces produits, la présence des substances caractéristiques des deux espèces. En outre, la quantité de ces substances est proportionnelle aux quantités relatives des composants azotés de chacun de ces laits.

b) Produits fromagers obtenus par mélange de fromage de vache et de fromage brebis.

Nous avons, pour des raisons pratiques, étudié le problème des mélanges sous cet aspect : mélanges de fromages affinés, Roquefort et Bleu. Nous les avons examinés par la méthode électrophorétique. Les figures 14 et 15 , sont les illustrations de l'une de ces études: mélanges de Roquefort et Bleu dans les proportions de 20/80, $40 / 60,60 / 40,80 / 20$. Ces fromages avaient des taux de protéines totales sensiblement identiques et un coefficient de protéolyse voisin: matières azotées solubles Roquefort: 51,4 p. 100 ; Bleu : 52,2 p. 100. 


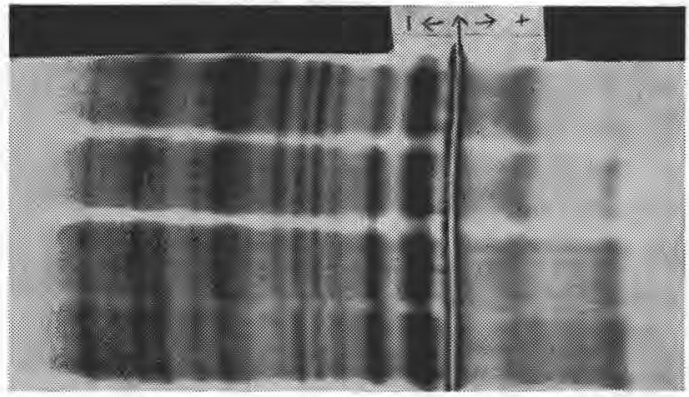

4. Roquefort/Bleu $80 / 20$

$\begin{array}{lll}3 . & \text { " } & 60 / 40 \\ 2 . & \text { " } 40 / 60 \\ 1 . & \quad \text { " } 20 / 80\end{array}$

Fig. 14.

Sur ces électrophorèses et électrophorégrammes, on remarque la présence des protéines caractéristiques de chaque catégorie de fromage. Pour l'identification de l'adultération caractère vache, nous recherchons la présence des bandes électrophorétiques $\mathrm{Rx} 0,20$, 1,21 et $-0,60$. Ces composants des fromages apparaissent sur le spectre proportionnellement au taux des mélanges, d'où possibilité d'évaluation.

\section{Discussion.}

Nous avons, par exemple, reporté sur graphique la relation quantité de fromage vache et densité optique de la substance $\mathrm{Rx} 0,20$. Le tracé de la droite-abaque (fig. 16), permet théoriquement d'évaluer une adultération.

En réalité, la teneur absolue de matières azotées et le niveau de protéolyse sont deux facteurs fluctuants. Il convient de considérer le principe du dosage photométrique mentionné comme une évaluation approchée. En fait, dans les limites de l'affinage normal 40 à 60 p. 100 de matières azotées solubles - la relation paraît assez bonne.

Pratiquement, la méthode permet d'identifier l'adultération à partir de 20 p. 100. Il semble possible, dans beaucoup de cas, de l'évaluer à partir de 10 p. 100.

\section{2) Mélanges de fromages affinés avec fromages peu affinés.}

Toutes les possibilités peuvent être ici envisagées. Nous avons $v u$, en deuxième partie, que les proportions des substances présentes variaient en fonction du taux d'affinage : prépondérance des caséines intactes dans le fromage peu affiné, prépondérance des caséines modifiées à partir d'un certain niveau de protéolyse. Nous avons envisagé l'éventualité de tels mélanges: par exemple, Roquefort affiné et Bleu de vache en début d'affinage. 


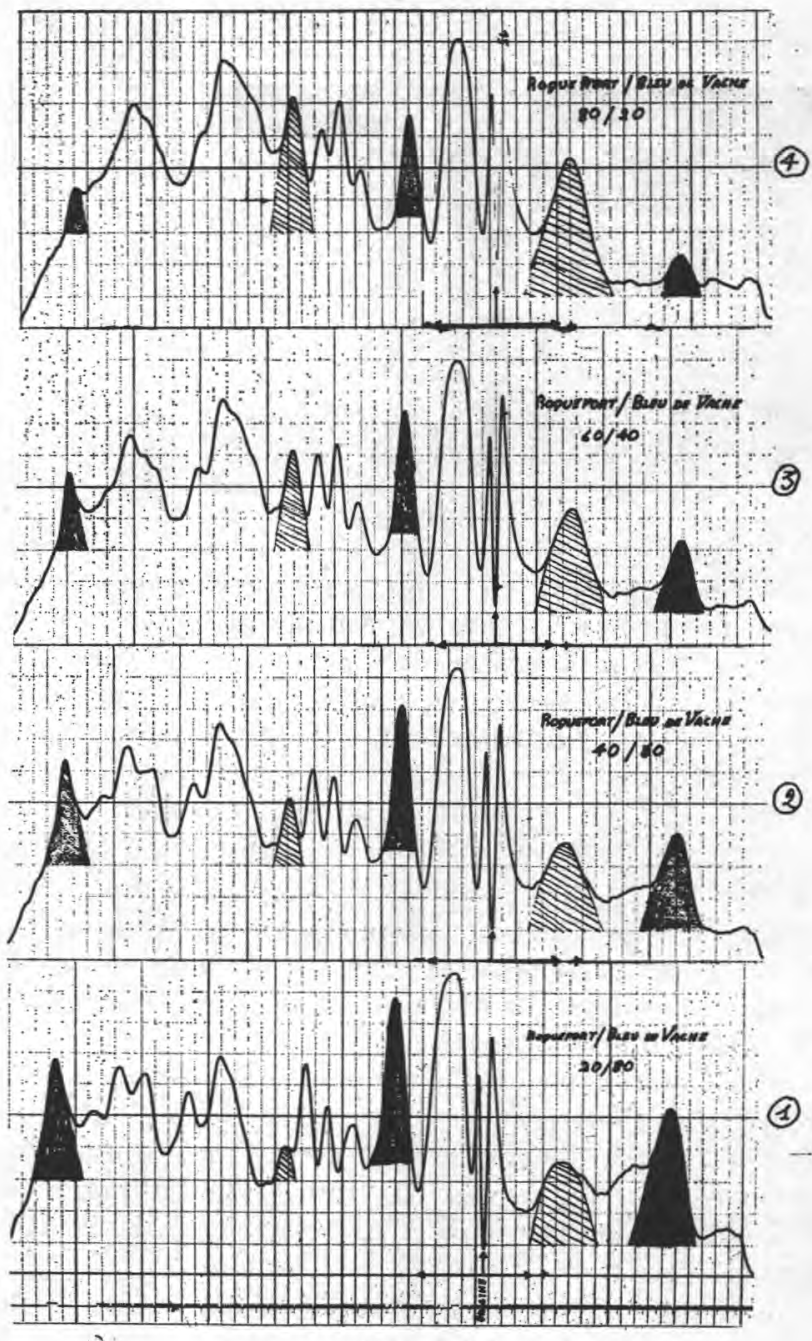

Fig. 15. - Diagrammes d'électrophorèses de mélanges : Roquefort/Bleu.

Pour un mélange à 50 p. 100 de cette nature, on observe :

- Une quantité importante de caséines $\alpha_{\zeta}$ vache $R x 1,10$;

- Une quantité importante de caséines $\alpha_{5}$ modifiées $R \times 1,21$;

- en faible proportion des caséines modifiées $\mathrm{Rx} 0,06$ et 0,20 .

Il est, on le voit, assez facile d'identifier de tels mélanges, mais il est plus difficile de l'évaluer de façon précise. 


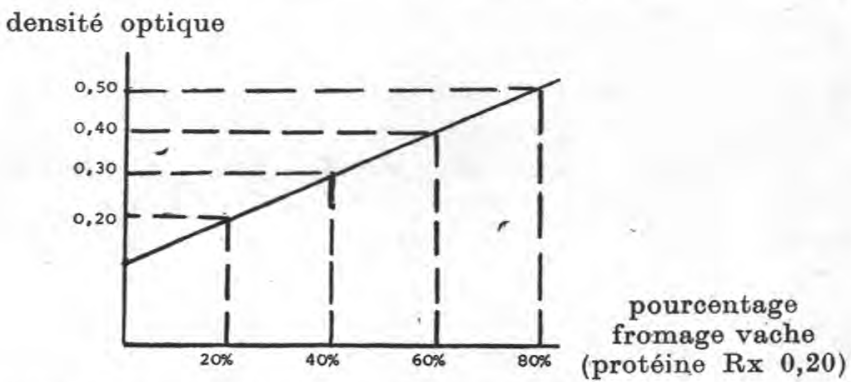

Fig. 16. - Evaluation du pourcentage du fromage de vache d'après la vale ur densitométrique de la protéine $R \times 0,20$.

\section{3) Fromages fondus - Dressings.}

L'identification des composants de ces produits se rapporte à ce qui a été dit dans les paragraphes précédents. Il convient de remarquer que les fabrications de tels produits se présentent sous de multiples aspects, et que les fromages qui entrent dans leur composition peuvent donner lieu à toutes sortes de mélanges.

L'identification et l'évaluation de ces mélanges sont délicates, mais l'expérience nous a appris qu'elles étaient possibles par électrophorèse. On repère les protéines et on détermine les adultérations éventuelles dans les mêmes conditions que dans les extraits de fromage.

\section{Conclusions}

De l'ensemble des observations recueillies au cours de cette étude, nous croyons pouvoir retenir les enseignements suivants:

1) Le procédé d'électrophorèse sur gel de polyacrylamide paraît être remarquablement adapté à la séparation des protéines du lait et du fromage. Il présente, en plus, le grand avantage d'être simple et pratique.

2) L'électrophorèse sur gel, appliquée aux protéines du lait de femme, de vache, de brebis et de chèvre et spécialement aux caséines, devient une méthode d'identification de l'espèce animale dont elles proviennent.

3) L'électrophorèse sur gel, appliquée aux protéines du fromage - caséines vraies et caséines modifiées - fournit également une méthode d'identification de leur lait d'origine. Elle a donné des résultats satisfaisants pour l'identification du Roquefort (au lait de brebis) et des Bleus (au lait de vache).

4) Ce procédé devient un moyen efficace de détection des adultérations: lait de brebis avec lait de vache; lait de chèvre avec lait de vache; adultérations de lait de femme, etc. La détection est possible à partir de 2 p. 100 d'addition. 
5) Le problème des adultérations du fromage de Roquefort avec le fromage Bleu de vache, semble, dans les mêmes conditions, trouver une solution satisfaisante. Elle pourrait être étendue à d'autres catégories de fromage.

6) Grâce à son pouvoir de séparation des protéines, l'électrophorèse sur gel d'acrylamide fournit le moyen de perfectionner les procédés biologiques d'identification des laits et des fromages.

Nous remercions $M$. Kahane, professeur de Chimie Biologique à la Faculté des sciences de Montpellier, M. Mocquot, directeur de la Station centrale de recherches laitières et de technologie des produits animaux au C.N.R.Z. à Jouy-en-Josas, et M. Garnier, Mâ̂tre de recherches à la Station centrale de recherches laitières et de technologie des produits animaux au C.N.R.Z. à Jouy-en-Josas, qui nous ont fait bénéficier de leurs conseils pour la réalisation de ce travail.

Nous sommes également reconnaissants à notre collaboratrice, $M^{11}$ Marquès, de son assistance technique en la matière.

\section{RÉFÉRENCES}

[1] Zimmerman (M.), 1928. Kinderheilk, 45, 310, cité par Dutrheil (H.), 1959. 15e Congrès international de laiterie, Londres, 3, 1522.

[2] Freudenberg (E.), 1927. Kinderheilk, 43, 454, cité par Dutherl (H.).

[3] Alison (F.), 1951, Arch. Pediatrie, 8, 723.

[4] Carrie (J.), $1934 \mathrm{~J}$. Agr. Res.

[5] Bordet (J.), 1899, Ann. Inst. Pasteur, 13, 225.

[6] Fisch, 1939, Traité de l'immunité, Ed. Masson.

[7] Fordenoff (M.) et KoschucharofF (P.), 1939, Veterinaria Kiska, cité par DutheIL (H.).

[8] Legros (J.), Arch. Pediatrie, 4, 561.

[9] Solberg (P.) et HADLAND (G.), 1953, $15^{\mathrm{e}}$ Congrès international de laiterie, Londres, 3, 1287.

[10] Dutherl (H.), 1959, 15 e Congrès international de laiterie, Londres, 3, 1522 .

[11] NAIR (P.) et IYA (K.), 1962, Milchwissenschaft, 17, 477.

[11 bis] Prnto (F. C.), 1966, J. Dairy Res., 33, 129.

[12] Aldis (Ch.), 1965, Science du Lait, Ed. 2 S. E. P., p. 110.

[13] Biserte (G.), Breton (A.), Fontanne (G.), 1953, La Semaine des Hópitaux, 31, 73.

[14] Ambrosino (C.), Liberatori (J.), Ubertalle (A.), 1961, Ric. Sc. 31, (II-B) 141.

[15] Hilpert (H.) et Enkelmann (D.), 1963, Milchwissenschaft, 18, 26.

[16] LiNDQwist (B.) et Storgards (T.), 1959, Congrès international de laiterie, Londres, 4, 679.

[17]. Aschaffenburg (R.), 1964, Bioch. Biophy. Acta, 82, 186.

[18] WaKe (R.) et Baldwin (R.), 1961, Bio. et Biophy. Acta, 47, 225.

[19] Rowland (S.), 1938, J. Dairy Res., 9, 42.

[20] AschaffenbuRg (R.), et Drewry (J.), 1959, 15 e Congrès international de laiterie, Londres, 1, 631.

[21] Zittle (C. A.) et Custer (J. H.), 1963, J. Dairy Sc., 46, 1183.

[22] Zittle (C. A.) et Custer (J. H.), 1966, J. Dairy Sc., 9, 288.

Erratum : Pour l'ensemble de la publication, les signes électriques des figures (électrophorèse) ont été inversés. Le lecteur rectifiera en conséquence. 